\title{
REVISIÓN DEL PROTOCOLO PARA EL TRATAMIENTO DE LA RETINOPATÍA DIABÉTICA
}

\author{
REVISION OF THE PROTOCOL FOR THE TREATMENT \\ OF DIABETIC RETINOPATHY
}

\author{
PAREJA-RÍOS A ${ }^{1}$, SERRANO-GARCÍA M², QUIJADA-FUMERO E ${ }^{1}$, MARRERO MD ${ }^{3}$, \\ CABRERA-LÓPEZ $\mathrm{F}^{4}$, ABREU-REYES $\mathrm{P}^{5}$, CARDONA-GUERRA $\mathrm{P}^{6}$, REYES-RODRÍGUEZ $\mathrm{M}^{7}$
}

\begin{abstract}
RESUMEN
Se presentan unas directrices generales con el objetivo de proporcionar una orientación en el manejo de la retinopatía diabética $(\mathrm{RD})$ en un momento en el que han aparecido numerosas alternativas terapéuticas cuyo papel aún no está suficientemente definido. Este protocolo está dirigido no a retinólogos expertos sino a oftalmólogos generales que precisen una guía práctica y actualizada de una patología tan prevalente como la RD.

En este documento se revisan las distintas opciones terapéuticas disponibles y su indicación más aceptada según el grado de retinopatía diabética que presente el paciente. Se plantea así que hacer con una retinopatía diabética no proliferativa (RDNP) leve, moderada (ambas control por su oftalmólogo de zona) y severa (en casos muy seleccionados puede considerarse la realización de una panfotocoagulación-PFC-). Los pacientes con retinopatía diabética proliferativa (RDP) serán tratados en los centros hospitalarios (PFC/fármacos antiangiogénicos/ciru-
\end{abstract}

\begin{abstract}
We present general guidelines to help us with the treatment of diabetic retinopathy (DR) at a time when numerous therapeutic alternatives have been developed although their role has not yet been adequately defined. This protocol is not directed at experienced retinologists but rather at general ophthalmologists who require a practical and up to date guide of a pathology as prevalent as RD.

The different therapeutic options available, and their most accepted indications depending on the degree of diabetic retinopathy that patients have, are reviewed. We propose what to do in cases of mild, moderate and severe non-proliferative diabetic retinopathy as well as in cases of proliferative diabetic retinopathy (panphotocoagulation/antiangiogenic drugs/vitreorretinal surgery). The treatment of diabetic macular edema depending on its angiographic and topographic characteristics is also discussed.

The importance of metabolic control of the patient is stressed (tight glycemic control, control
\end{abstract}

\footnotetext{
Recibido: 2/6/07. Aceptado: 18/2/09.

Hospital Universitario de Canarias. Tenerife. España.

1 Doctor en Medicina. Médico Adjunto de la Sección de Retina. Hospital Universitario de Canarias.

2 Doctor en Medicina. Jefe de Sección de Retina. Hospital Universitario de Canarias.

3 Doctor en Medicina. Médico Adjunto de la Sección de Retina. Hospital Negrín G.C.

4 Doctor en Medicina. Médico Adjunto de Retina. Hospital Insular G.C.

5 Doctor en Medicina. Jefe de Servicio del Hospital Universitario Nuestra Señora de La Candelaria.

6 Doctor en Medicina. Jefe de Servicio del Hospital Insular de Las Palmas de G.C.

7 Doctor en Medicina. Médico Adjunto de la Sección de Retina. Hospital Negrí G.C.

Comunicación presentada parcialmente en el XXXV Congreso de la Sociedad Canaria de Oftalmología.

Correspondencia:

Alicia Pareja Ríos

Hospital Universitario de Canarias

C/. Ofra, s/n

38320 La Laguna (Tenerife)

España

E-mail: aparejar@gmail.com
} 
gía vítreorretiniana -CVR-) hasta que sea controlado su proceso. Se discute asimismo el tratamiento del edema macular (EM) diabético según sus características angiográficas y topográficas.

Se hace hincapié en la importancia del control metabólico del paciente (optimizar el control glucémico, de su hipertensión arterial y de la dislipemia) como tratamiento necesario y coadyuvante de su RD.

Esta propuesta terapéutica ha sido ampliamente discutida por retinólogos de los cuatro grandes hospitales de Canarias por lo que se trata de un texto consensuado basado en la bibliografía científica actual.

Palabras clave: Retinopatía diabética (RD), edema macular diabético (DME), tomografía de coherencia óptica (OCT), vitrectomía, fármacos antiangiogénicos intravítreos. of arterial hypertension and dyslipemia) in aiding the treatment of diabetic retinopathy.

This therapeutic proposal has been discussed widely by retinologists from the four largest hospitals in the Canary Islands, and is therefore an agreed text based on recent scientific literature (Arch Soc Esp Oftalmol 2009; 84: 65-74).

Key words: Diabetic retinopathy, Diabetic macular edema, optic coherence tomography, vitrectomy, intravitreal antiangiogenic drugs.

\section{MANEJO DE LA RETINOPATÍA DIABÉTICA}

Este protocolo sugiere líneas de actuación para diversos supuestos diabéticos, pero no pretende establecer criterios de obligado cumplimiento, ni eximir al oftalmólogo de su responsabilidad de reflexionar ante un caso concreto y actuar según su buen criterio profesional. Además en modo alguno limita o vincula la libertad del oftalmólogo en su toma de decisiones para el tratamiento de un paciente determinado. Puede así optar por otra pauta distinta, dentro de las técnicas normales requeridas, si entiende que, según su experiencia, el resultado buscado exige otro tipo de terapia. El que dicha opción no esté contemplada en este documento como pauta de actuación recomendada, no puede considerarse en modo alguno como una mala praxis profesional.

Para adaptarlo al sistema sanitario al que se pertenezca puede ser necesario variar algunos aspectos del mismo, por ejemplo, en nuestro medio no existen aparatos de láser en los ambulatorios por lo que el tratamiento de la retinopatía diabética proliferativa y el edema macular debe ser necesariamente hospitalario. Las comunidades sanitarias en las que los ambulatorios estén dotados de este tipo de aparataje derivarán fundamentalmente a los hospitales los casos que sean quirúrgicos.

Para establecer las pautas de tratamiento nos basaremos en las siguientes clasificaciones.

\section{CLASIFICACIÓN CLÍNICA INTERNACIONAL DE LA RD (GDRPG)}

- Sin retinopatía diabética (RD) aparente: Ausencia de microaneurismas.

- RD no proliferativa (RDNP) Leve: Sólo microaneurismas (fig. 1).

- RDNP Moderada: Microaneurismas asociado a menos de 20 hemorragias $(\mathrm{H})$ intrarretinianas en cada uno de los cuatro cuadrantes (C), exudados duros (ED), «exudados» algodonosos (EA), arrosariamiento venoso (VB) en uno sólo C (fig. 2).

- RDNP Severa: Regla del 4 - 2 - 1.

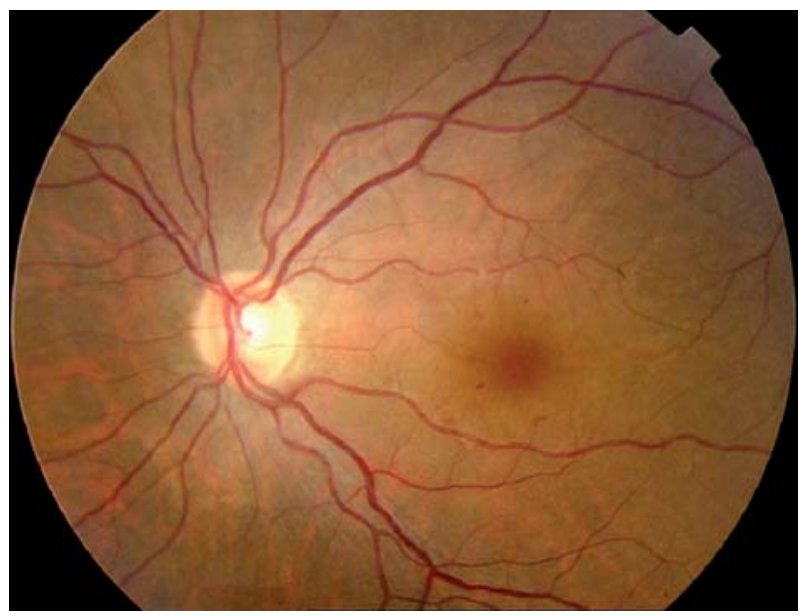

Fig. 1: RDNP leve. 


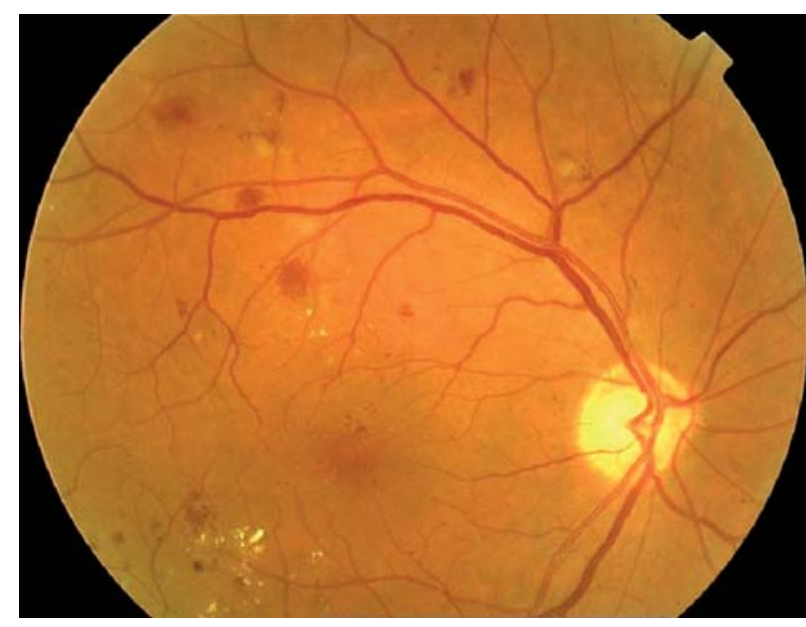

Fig. 2: RDNP moderada.

- Microaneurismas $+\mathrm{H}$ intraretinianas severas (>20) en cada uno de los cuatro $\mathrm{C}$ o arrosariamiento venoso en $\geq$ dos $\mathrm{C}$ o anomalías microvasculares intrarretinianas (AMIR)en $\geq$ un C (fig. 3).

$\mathrm{Si}$ en lugar de «0» es «y» se trataría de una RDNP muy severa.

RDP: Neovasos (NV) y/o hemorragia prerretiniana o vítrea (fig. 4).

\section{CLASIFICACIÓN EDEMA MACULAR (ETDRS) (1985)}

- Sin edema macular.

- Con edema macular (EM): engrosamiento retiniano dentro de un diámetro de disco (DD) del centro de la mácula (no es EM la difusión de fluoresce-

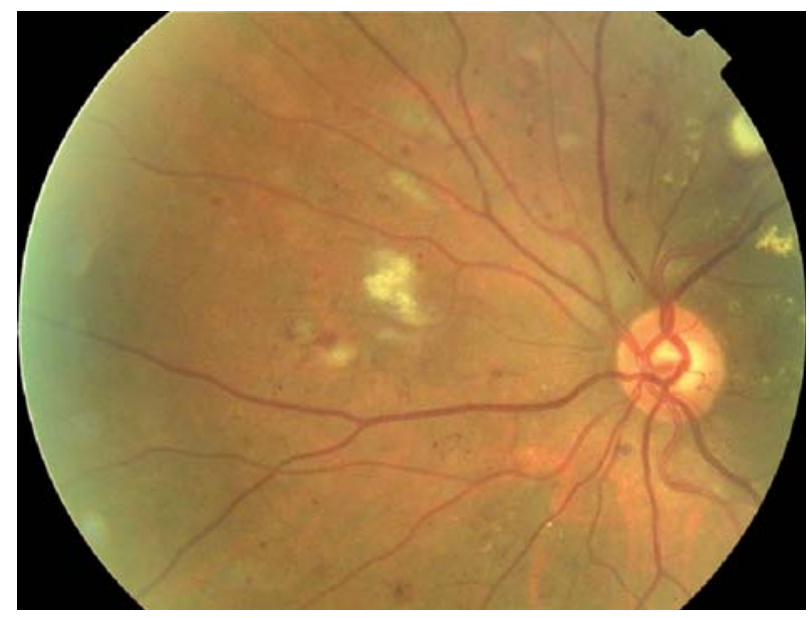

Fig. 3: RDNP severa.

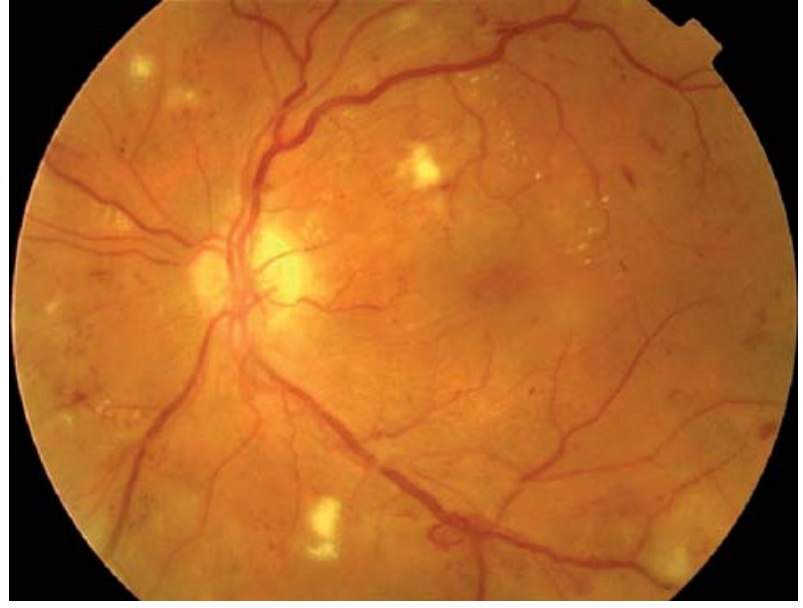

Fig. 4: RDP.

ína en la AFG si no existe engrosamiento). En el ETDRS también se clasificó como EM cuando existían exudados duros dentro de un DD del centro.

- Con edema macular clínicamente significativo (EMCS) (figs. 5-7):

- Engrosamiento retiniano dentro de $500 \mu$ del centro.

- Exudados duros dentro de $500 \mu$ del centro si se asocia a un engrosamiento de retina adyacente.

- Un área de engrosamiento retiniano del tamaño de al menos un área del disco, parte de la cual está a menos de un DD del centro.

- Fijarse que la agudeza visual NO forma parte de la definición de edema macular y que ésta puede ir de 1.2 a percepción de luz (PL), ambas incluidas, y presentar EMCS.

\section{CLASIFICACIÓN ANGIOGRÁFICA DEL EM}

- EM focal: áreas bien definidas de difusión procedente de microaneurismas individuales o asociados (fig. 5).

- EM multifocal (que frecuentemente se confunde con el EM difuso) (fig. 6).

- EM difuso: se asocia con frecuencia al edema macular quístico (EMQ). Se ha definido como un engrosamiento retiniano $\geq 2$ diámetros de papila con afectación de alguna porción de la zona avascular foveal. Suele ser bilateral. Clínicamente se traduce en un área de edema poco definida, con pocos microaneurismas y pocos exudados duros, con más o menos isquemia en la angiografía (AFG), brillos, 


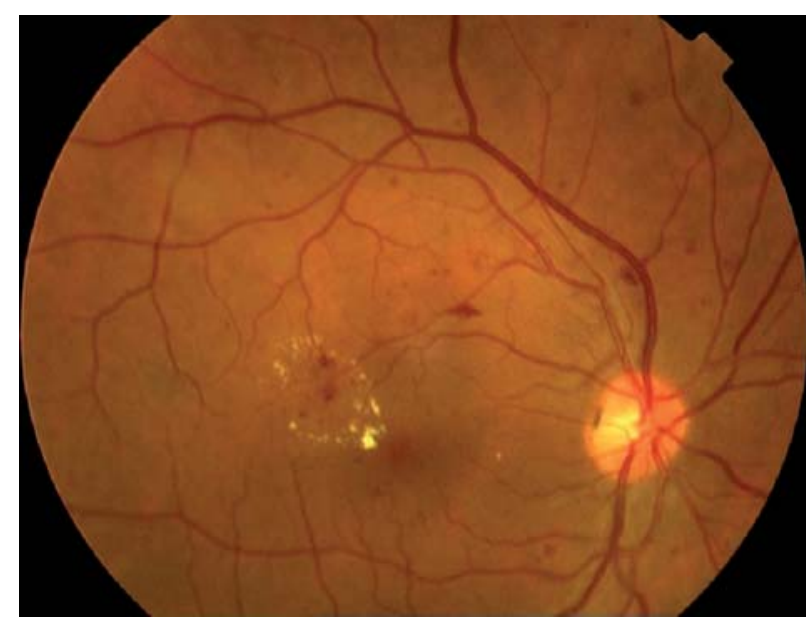

Fig. 5: EMCS focal.

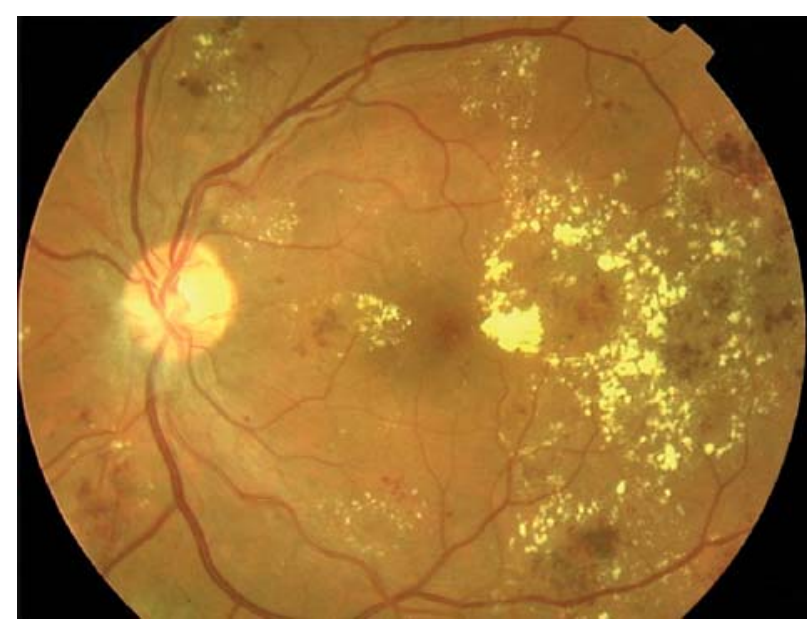

Fig. 6: EMCS multifocal.

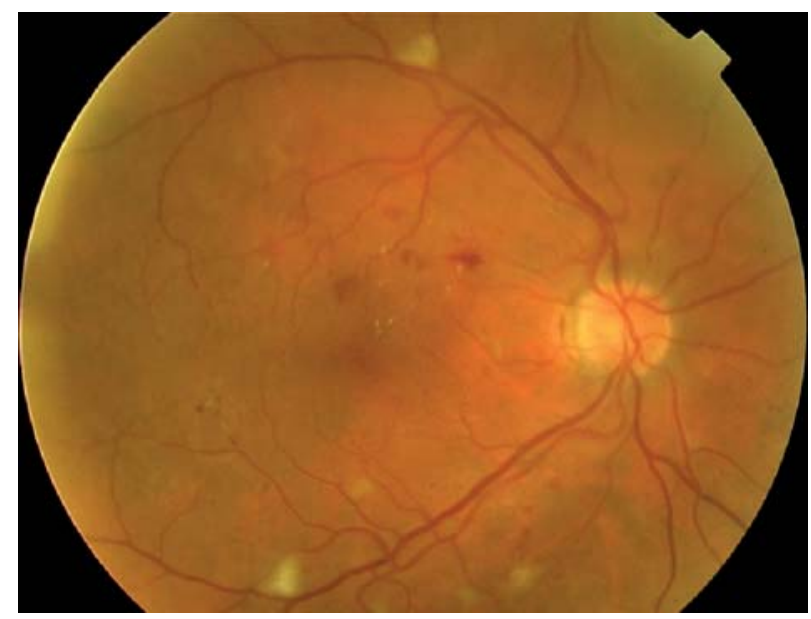

Fig. 7: EMCS difuso. opacidad de la hialoides posterior y con o sin membranas epirretinianas (fig. 7).

- EM mixto.

\section{MANEJO DE LA RETINOPATIA DIABÉTICA}

\section{Establecimiento de la primera exploración oftálmica}

- Diabetes tipo 1: la primera revisión se hará a los 3-5 años del diagnóstico. En caso de presentarse algún signo de retinopatía se aconseja mantener el control ocular en los centros hospitalarios, en base al alto grado de complicaciones y el carácter agresivo de las mismas.

- Diabetes tipo 2: la primera revisión se hará en el momento del diagnóstico aconsejándose controles anuales o bianuales posteriores hasta la aparición de algún grado de retinopatía. Al detectarse ésta, los criterios aconsejados son expuestos según el grado de afectación, según la existencia o no de edema, y según la presencia de complicaciones secundarias a una retinopatía avanzada.

\section{Controles oftálmicos posteriores}

Para establecer la frecuencia de las revisiones posteriores nos referiremos primero a los distintos grados de retinopatía y luego al estado de la mácula. En cuanto a la periodicidad de las revisiones primará el criterio de las más cercanas, por ejemplo si un paciente con RDNP leve, cuyo control aconsejado es anual, presenta también un EMCS asociado la periodicidad de las visitas las dictará este último (por ejemplo cada 3 meses).

En todos los estadios de la RD es esencial el control endocrinológico fundamentalmente de: glucemia, hipertensión arterial (HTA), sobrepeso, lípidos, estado cardíaco y renal.

\section{Sin retinopatía diabética aparente}

Control oftalmológico anual o bianual.

\section{Con RDNP leve (fig. 1)}

Control oftalmológico anual. 
Adelantar control en caso de: gran cantidad de microaneurismas, que amenacen fóvea..., primera exploración de paciente muy mal controlado, paso reciente de antidiabéticos orales a insulina, pacientes embarazadas (se hará un control en cada trimestre y luego cada 6 meses durante el primer año tras el parto).

\section{Con RDNP moderada (fig. 2)}

Control oftalmológico semestral.

No precisa AFG.

No precisa panfotocoagulación (PFC) dado que el riesgo de progresión a RDP al año oscila entre un $1-8 \%$.

\section{Con RDNP severa (fig. 3)}

Las RDNP severas son casos que deben ser considerados de alto riesgo, dada la probabilidad de progresión a RDP que se sitúa entorno al 50,2\% en un año y a RDP con CAR que está alrededor de un $14,6 \%$ (1).

DM 2: control oftalmológico cada 2-4 meses.

DM 1: control oftalmológico cada 2-4 meses.

Considerar PFC temprana en aquellos pacientes con mayor riesgo de progresar: DM 2 con mal control metabólico y pacientes que no cumplen las revi-
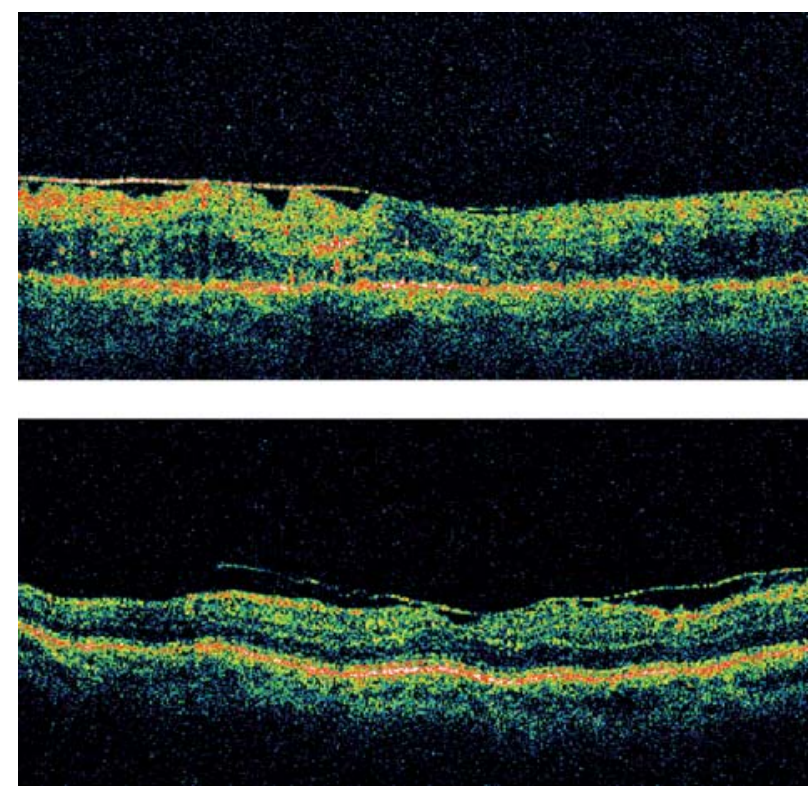

Fig. 8: Síndrome de tracción vítreomacular en OCT. siones de forma habitual, RDP en el otro ojo, previo a la cirugía de catarata, embarazo o deseo del mismo, isquemias AFG generalizadas.

En estos casos el orden de fotocoagulación que se aconseja es realizar un cuadrante por sesión comenzando por el nasal o el inferior y finalizando por el temporal (fig. 9). En cuanto a la frecuencia, se dará una sesión cada 2-3 semanas (si se puede esperar, mejor cada 3 semanas) ya que la mácula no se recupera en 1 sola semana (2).

\section{RDP y medios transparentes (fig. 4)}

PFC en un mes o mes y medio: sesiones de 300500 impactos (si existe tracción hacer sesiones de 200-300 impactos). Revisiones cada 3-6 meses.

Si no responde: opciones: 1. antiangiogénicos $(*)$ : cada 4-6 semanas. 2. Cirugía retinovítrea (CRV).

\section{Casos especiales}

- Con adherencias vitreoretinianas extensas: CRV con PFC -se puede usar antiangiogénicos previos (indicación en estudio*) + CRV antes del 3. ${ }^{\text {er }}$ día + Antiangiogénicos (indicación en estudio*)-.

- Papilopatía diabética traccional: CRV.

- Maculopatía traccional: CRV.

- Rubeosis iridiana: PFC extensa.

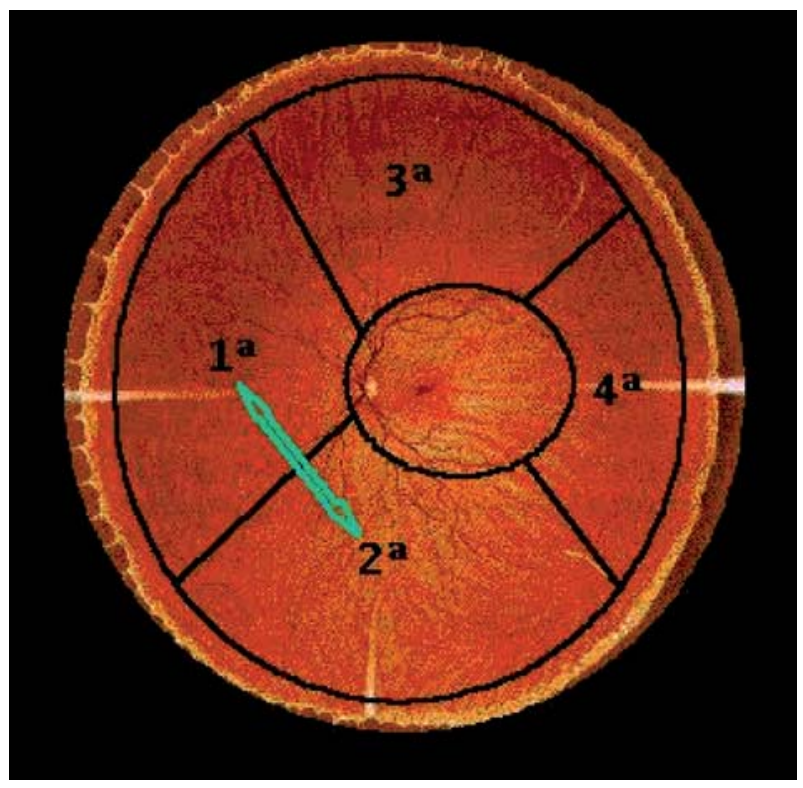

Fig. 9: Secuencia de fotocoagulación por cuadrantes. 


\section{RDP con hemovítreo}

Recordar que limitar la actividad de los pacientes diabéticos con RDP no es efectivo para prevenir el hemovítreo. De existir el sangrado, tampoco el prescribir reposo resulta eficaz. Por ello, ante los dos supuestos, el paciente puede realizar la actividad que le permita su limitada visión.

- SI DR regmatogeno o mixto: CRV urgente.

- SI no DR (paciente vitrectomizado con hemovítreo: riesgo de proliferación anterior): Esperar 2 meses. Controles de AV / Ecográficos cada 15 días.

Cirugía diferida (2-3 meses) de no haber mejoría.

Antiangiogénicos (*) en caso de: hemorragia persistente o hemorragia muy severa. Se inyectarán cada 4 semanas manteniendo una estrecha vigilancia por riesgo de desprendimiento de retina traccional (DRT). Si no mejora: CRV tras antiangiogénicos*: no más allá del $3 .^{\text {er }}$ día.

\section{RDP con hemorragia premacular-retrohialoidea}

CRV precoz, preferiblemente antes de un mes.

\section{RDP con hemovítreo recurrente tras $C R V$}

Sangrado inmediato (3)

Ocurre a partir del tejido fibrovascular residual disecado durante la vitrectomía o dispersión de sangre residual. La gran mayoría se resuelven espontáneamente en días o semanas (podemos decirle al paciente que permanezca con la cabeza ligeramente elevada) esperar 1-2 meses con control de AV y ecográfico. Si no desaparece: vitrectomía para restablecer la visión y eliminar los productos que puedan estimular la proliferación.

CRV en 2-4 meses [antiangiogénico preoperatorio $(*)]$.

Puede intentarse el intercambio sangre-aire.

Opción antiangiogénica: Antiangiogénicos(*): cada 4-6 semanas.

Control ecográfico cada 15 días.

Si mejora: completar PFC/crioterapia periferia extrema.
Sangrado tardío

Aparece en un número significativo de casos (del $13 \%$ al $50 \%$ ) y puede tener distintas causas. Exponemos las dos más frecuentes.

- Sangrado del tejido proliferativo periférico presente en las esclereotomías: el origen de estos neovasos no es la retina anterior (como en la proliferación fibrovascular anterior) sino el cuerpo ciliar. Este tejido fibrovascular se puede extender hacia vítreo anterior dada su proximidad. Se asocia a incarceración del vítreo en las esclereotomías. El diagnóstico puede hacerse por depresión escleral. A veces puede existir un vaso centinela epiescleral entrando en la esclerotomía (aunque su existencia no asegura que exista tejido fibroproliferativo en la misma).

El tratamiento consistirá en una nueva CRV extrayendo este tejido pudiéndose dejar gas como tamponador.

- Proliferación fibrovascular anterior (4). En estos casos, el hemovítreo ocurre entre 1 y 7 meses después de la cirugía retinovítrea. Se trata de una proliferación neovascular que se origina en la retina periférica y se extiende a través de la hialoides anterior hacia la cara posterior de la cápsula cristaliniana. El examen con depresión escleral y la biomicroscopía indirecta revelan la neovascularización, a veces aparece también rubeosis en iris. En la ecografía pueden detectarse bandas de tejido engrosado desde la retina periférica al cuerpo ciliar y superficie posterior del iris, desprendimientos traccionales de la retina anterior y del cuerpo ciliar (que se asocian con frecuencia a hipotonía). Como su tratamiento es desalentador lo más importante es la prevención sobre todo en pacientes con factores de riesgo de padecerla: como jóvenes con DM tipo 1, isquemia retiniana severa, DR traccional y/o mixto sobre todo si se les ha colocado cerclaje extraescleral, múltiples cirugías, RDP no tratada o que no responde a la PFC, RDP con proliferación posterior fibrovascular extensa, rubeosis iridis postoperatorias, hemovítreos recurrentes...

En éstos hay que hacer PFC agresivas. Si aún así evolucionan, lo indicado es una CRV extrayendo cuidadosamente el vítreo anterior (para lo que puede ser necesario una lensectomía) y realizar una fotocoagulación anterior extensa.

Si la proliferación fibrovascular anterior ya está presente hay que detectarla lo más precozmente posible, antes de que ocurra un DR traccional de 
retina y/o de cuerpo ciliar y se formen membranas ciclíticas. Se debe hacer una cirugía retinovítrea seguida de lensectomía extrayendo la cápsula posterior del cristalino, disección de la base del vítreo, endodiatermia de neovasos y endofotocoagulación lo más extensa posible (evitar la crioterapia ya que ésta puede causar contracción del tejido fibrótico y DR periférico). Si existen DR traccionales periféricos de retina y/o del cuerpo ciliar con frecuencia se necesita hacer retinotomías periféricas y usar aceite de silicona para conservar el globo ocular.

\section{RDP con desprendimiento de retina}

Traccional que afecte o amenace fóvea: CRV+TAIV $(*)$ como manipulador+tamponador electivo (gas/aceitie de silicona).

Regmatógeno/mixto: CRV $<48 \mathrm{hr}+$ aceite de silicona.

\section{RDP con glaucoma neovascular}

1. ${ }^{\text {er }}$ estadio: colirios de Atropina, antiglaucomatosos: todos: exceptos pilocarpina.

Secuencia: Brimomidina, Betabloqueantes, Inhibidores de anhidrasa carbónica: tópicos o sistémicos. Los análogos de las prostaglandinas son discutibles (está bloqueado el acceso a la vía uveoescleral por el tejido fibrovascular en el ángulo y aumenta la inflamación). Terapia Antiangiogénica (*). PFC: completarla al máximo.

2. ${ }^{\circ}$ estadio: sin respuesta en la PIO. Trabeculectomía con antimetabolitos / Implantes valvulares / Cicloablación. Fenolización (si ojo en amaurosis) / Evisceración / Enucleación.

\section{Catarata madura}

Los diabéticos $<65$ años tienen entre 3 y 4 veces mayor riesgo de desarrollar catarata sobre todo cortical y subcapsular posterior- que la población no diabética (en los grupos más jóvenes el riesgo aumenta hasta 25 veces). A partir de los 65 años el riesgo se iguala.

Siempre que la catarata permita visualizar el fondo ocular, o tratar con láser la progresión de la retinopatía, la postura será expectante. Cuando se supere estos límites, la facoemulsificación esta indi- cada. Tras la cirugía, el paciente debe ser fotocoagulado si procede o vigilado por si el trauma quirúrgico induzca la progresión de la retinopatía.

\section{EDEMA MACULAR DIABÉTICO}

\section{Valoración previa a la actuación terapéutica}

Si en la retinopatía diabética, el control sistémico es indiscutible, en el edema macular esto resulta aún más evidente. Se debe insistir en el control de la glucemia, HgbA1C, presión arterial, sobrepeso y lípidos. Enviar al endocrino/internista/nefrólogo si es necesario. Es realmente asombroso cómo mejoran algunas retinopatías diabéticas al mejorar el control metabólico.

Si existe mal control metabólico puede posponerse el tratamiento del edema macular hasta que éste mejore.

La valoración del edema debe incluir siempre mejor agudeza visual corregida, examen biomicroscópico, retinografía y OCT. La AFG puede no ser necesaria en algunos casos claros de circinadas donde se ve el origen de la exudación.

\section{Opciones terapéuticas}

\section{Edema macular clinicamente significativo (EMCS)} focal o multifocal (figs. 5 y 6 )

El tratamiento de elección de este tipo de edema continúa siendo la fotocoagulación focal.

En casos excepcionales en los que el engrosamiento macular central dificulte la laserterapia focal, que suele corresponder a valores de OCT superiores a 400 micras, podría proponerse corticoterapiav local1/antiVegf y una vez se logra la reducción del edema, se procedería a las 3-4 semanas a la fotocoagulación focal.

En caso de tracción vítreo-macular se procedería a CRV (6-9) (figs. 10 y 11) con o sin disección de MLI (10-12) y TAIV(*). La eficacia de la disección de la membrana limitante interna está en estudio y no se disponen de datos concluyentes sobre su impacto en la evolución del EM.

Si se produce una mejoría estable el paciente pasa a control por su oftalmólogo. Si no mejora sería tratado como un edema macular focal o multifocal sin tracción (gráfica 1). 


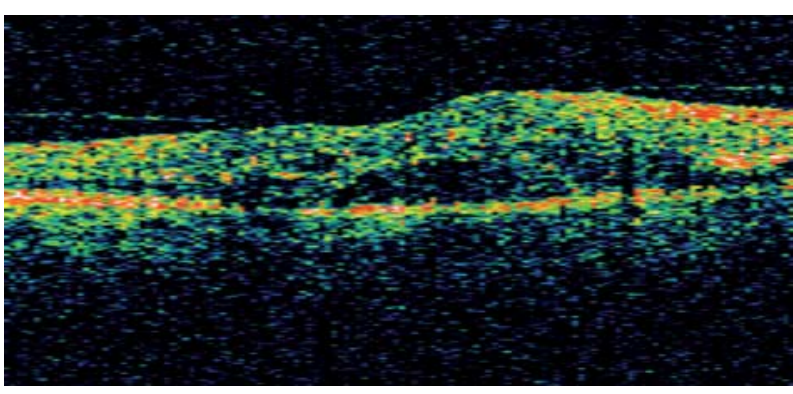

Fig. 10: Edema macular con síndrome de tracción vítreo-macular.

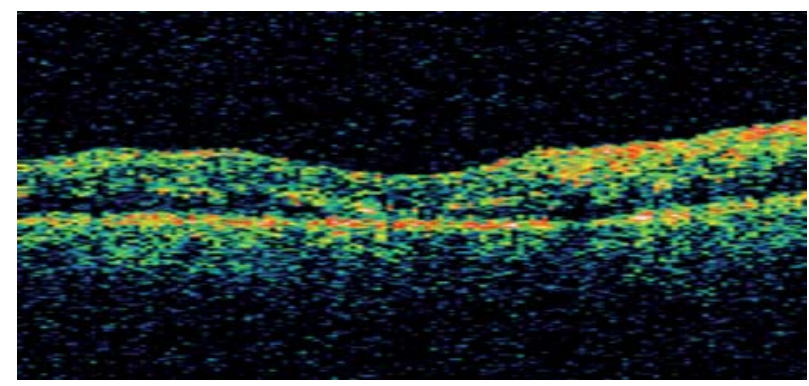

Fig. 11: El mismo caso tras cirugía retinovítrea.

\section{EMCS difuso (fig. 7)}

Cuando existe un síndrome de tracción vítreorretiniana la indicación terapéutica es la CRV con hialodectomía siendo opcional la disección de la membrana limitante interna y el uso final de TAIV (*) $(13,14)$. Si se produce una mejoría estable el paciente pasa a control por su oftalmólogo. Si no mejora sería tratado como un edema macular difuso sin tracción.

Si no existe tracción, la opción actualmente aceptada es el tratamiento con láser en rejilla modificada. En esta última se usan quemaduras menos intensas (gris suave) y más pequeñas $(50 \mu)$ y se trata los microaneurismas de forma directa pero sin intentar conseguir el cambio de coloración de los mismos. Asimismo trata zonas de engrosamiento retiniano y áreas de no perfusión que se crean en relación con el edema. Como los resultados de este tratamiento no son del todo satisfactorios existen algunas alternativas terapéuticas que, aunque existen numerosos indicios acerca de su efectividad, no están aún suficientemente probadas. Entre ellas nosotros nos inclinamos por la administración de una inyección intravítrea de triamcinolona(*) seguida, a las 2-3 semanas, de laserterapia en rejilla modificada. Si no

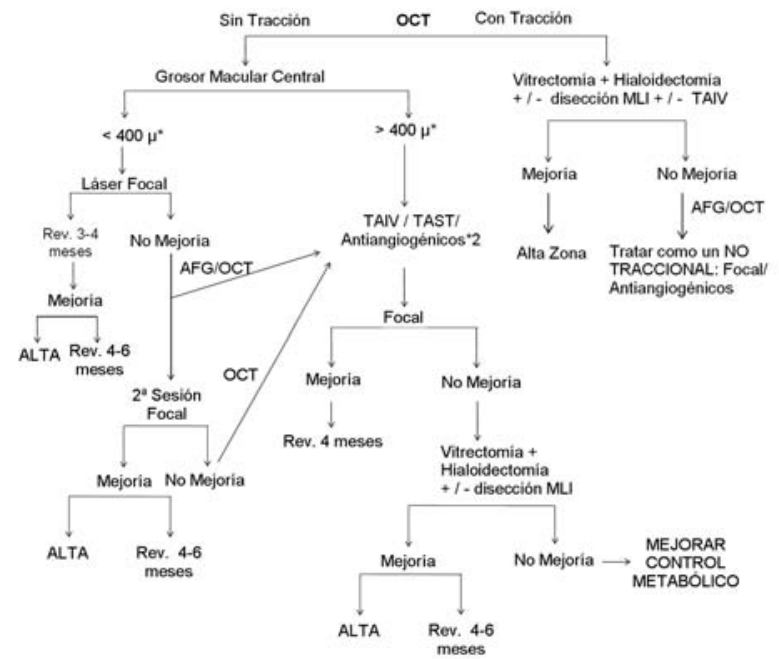

Gráfica 1: Algoritmo de tratamiento del edema macular focal y multifocal.

fuera posible el uso de la TAIV, se plantea como alternativa los antiangiogénicos intravítreos (15-21) o la corticoterapia subtenoniana (22). Cuando el edema se reduce intentaremos localizar las zonas de fugas más evidentes y actuar sobre ellas con láser.

$\mathrm{Si}$ se produce una mejoría duradera el paciente pasa a control por su oftalmólogo. Si no mejora se usarán antiangiogénicos (*) (23) bien el empleado

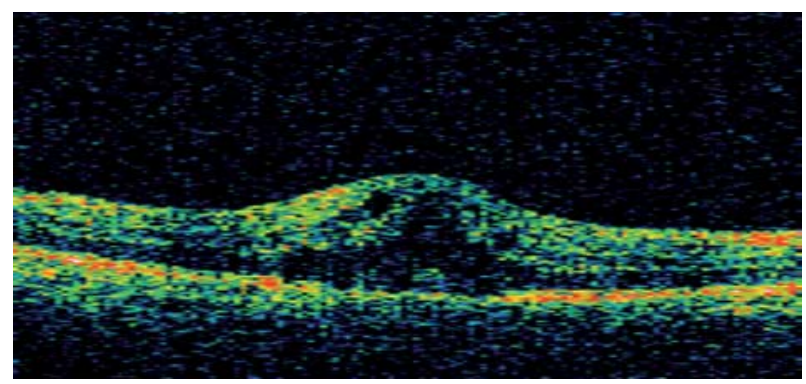

Fig. 12: Edema macular quístico.

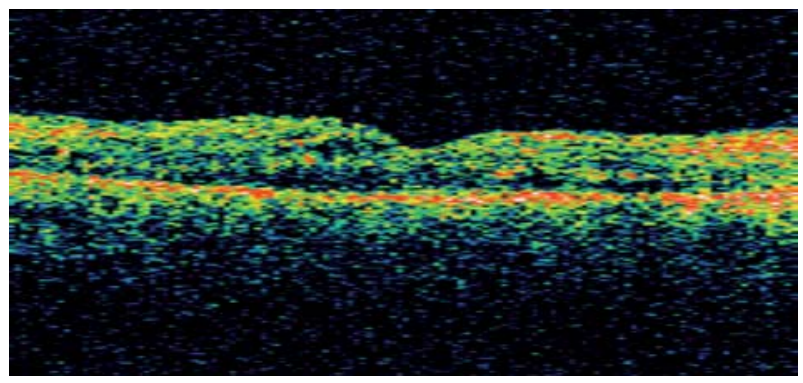

Fig. 13: El mismo EMQ tras TAIV. 


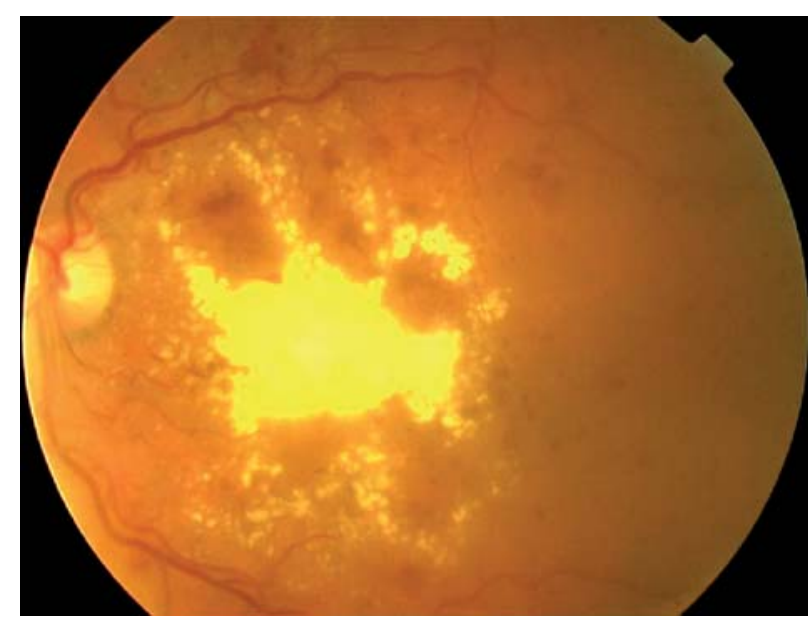

Fig. 14: EMCS con abundantes exudados duros maculares.

previamente $\mathrm{u}$ otros. Si no responden las opciones terapéuticas son muy reducidas y sólo en casos muy seleccionados se realizará cirugía retinovítrea $(24,25)$ (figs. 14 y 15$)$ ya que hay estudios que no encuentran mejoría (Figueroa MS, Contreras I, Noval S. Resultados y complicaciones de la vitrectomía vía pars plana en el edema macular diabético difuso no traccional. Madrid: XI Congreso de la Sociedad Española de Retina y Vítreo; 2007: 68.) (gráfica 2).

\section{Edema macular quístico (fig. 12)}

Ante un Edema Macular difuso Quístico (EMQ) con tracción vitreomacular se propone CRV sin disección de la membrana limitante interna (MLI)

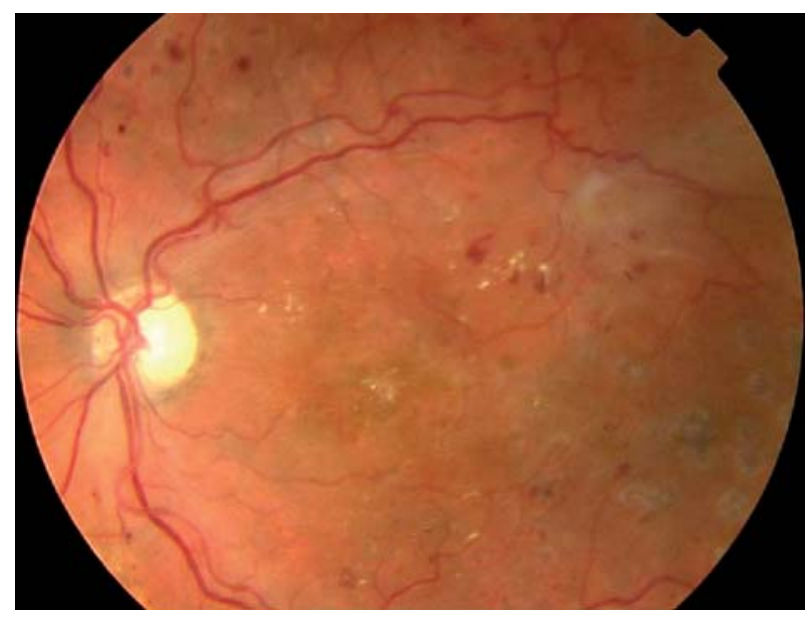

Fig. 15: El mismo edema tras CRV con pelaje de MLI.

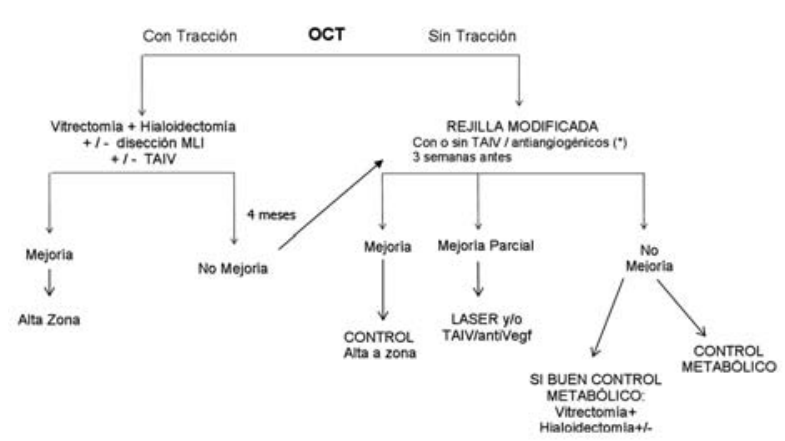

Gráfica 2: Algoritmo de tratamiento del edema macular difuso.

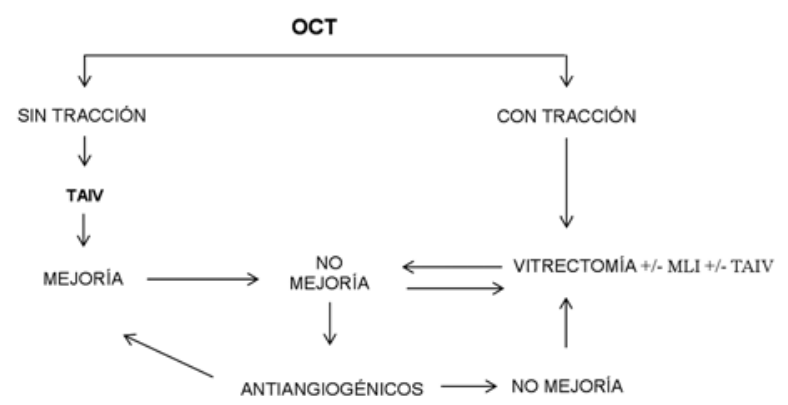

Gráfica 3: Algoritmo de tratamiento del edema macular difuso quístico.

en aquellos casos en que se desconozca el tiempo de evolución o que éste sea superior a 6 meses (por el riesgo a inducir un agujero macular). En casos de que sea inferior a 6 meses podría realizarse la disección de la MLI.

En los pacientes afectos de EMQ en los que no exista evidencia de tracción lo más indicado es la TAIV (*) seguido de forma opcional de antiangiogénicos y/o rejilla modificada (26) (gráfica 3) (figs. 12 y 13).

\section{BIBLIOGRAFÍA}

1. Verdaguer Tarradella J. Clasificación de la Retinopatía diabética. En: Alfaro V, Gómez-Ulla F, Quiroz-Mercado $H$, Figueroa MS, Villalba V SJ. Retinopatía diabética. Tratado médico quirúrgico. Madrid: MacLine SL; 2006; Cap 3: 43-50.

2. Shimura M, Yasuda K, Nakazawa T, Kano T, Ohta S, Tamai M. Quantifying alterations of macular thickness before and after panretinal photocoagulation in patients with severe diabetic retinopathy and good vision. Ophthalmology 2003; 110: 2386-2394. 
3. Arévalo F, Alfaro V, García RA, Fernández CF. Complicaciones de la vitrectomía en el paciente diabético. En: Alfaro V, Gómez-Ulla F, Quiroz-Mercado H, Figueroa MS, Villalba V SJ. Retinopatía diabética. Tratado médico quirúrgico. Madrid: MacLine SL; 2006; Cap 34: 445-462.

4. Berrocal MH. Proliferación fibrovascular hialoidea anterior. En: Alfaro V, Gómez-Ulla F, Quiroz-Mercado H, Figueroa MS, Villalba V SJ. Retinopatía diabética. Tratado médico quirúrgico. Madrid: MacLine SL; 2006; Cap 32: 425-429.

5. Diabetic Retinopathy Clinical Research Network. A randomized trial comparing intravitreal triamcinolone acetonide and focal/grid photocoagulation for diabetic macular edema. Ophthalmology 2008; 115: 1447-1449.

6. Cabrera López F, Cabrera Marrero B, Baeta Bayón L, Jerez Olivera E, Cardona Guerra P. Actualización en el diagnóstico y tratamiento del edema macular diabético. Arch Soc Can Oftalmol 2005; 16: 69-82.

7. Lewis H, Abrams GW, Blumenkranz MS, Campo RV. Vitrectomy for diabetic macular traction and edema associated with posterior hyaloidal traction. Ophthalmology 1992; 99: 753-759.

8. Pendergast SD, Hassan TS, Williams GA, Cox MS, Margherio RR, Ferrone PJ, et al. Vitrectomy for diffuse diabetic macular edema associated with a taut premacular posterior hyaloid. Am J Ophthalmol 2000; 130: 178-186.

9. Harbour JW, Smiddy WE, Flynn HW Jr, Rubsamen PE. Vitrectomy for diabetic macular edema associated with a thickened and taut posterior hyaloid membrane. Am J Ophthalmol 1996; 121: 405-413.

10. Gandorfer A, Messmer EM, Ulbig MW, Kampik A. Resolution of diabetic macular edema after surgical removal of the posterior hyaliod and the inner limiting membrane. Retina 2000; 20: 126-133.

11. Avic R, Karderli B, Avic B, Simsek S, Baykara M, Kahveci $Z$, et al. Pars plana vitrectomy and removal of the internal limiting membrane in the treatment of chronic macular oedema. Graefes Arch Clin Exp Ophthalmol 2004; 242: 845-852.

12. Recchia FM, Ruby AJ, Recchia CA. Pars plana vitrectomy with removal of the internal limiting membrane in the treatment of persistent diabetic macular edema. Am J Ophthalmol 2005; 139: 447-454.

13. David J, Browning CM, Powers F, Powers ME. Comparison of the magnitude and time course of macular thinning induced by different interventions for diabetic macular edema: implications for sequence of application. Ophthalmology 2006; 113: 1713-1719.

14. Yanyali A, Nohutcu AF, Horozoglu F, Celik E. Modified grid laser photocoagulation versus pars plana vitrectomy with internal limiting membrane removal in diabetic macular edema. Am J Ophthalmol 2005; 139: 795-801.
15. Avery RL, Pearlman J, Pieramici DJ, Rabena MD, Castellarin AA, Nasir MA, et al. Intravitreal bevacizumab (Avastin)in the treatment of proliferative diabetic retinopathy. Ophthalmology 2006; 113: 1695-1705.

16. Spaide RF, Fisher YL. Intravitreal bevacizumab (Avastin) treatment of proliferative diabetic retinopathy complicated by vitreous hemorrhage. Retina 2006; 26: 275-278.

17. Friedlander SM, Welch RM. Vanishing disc neovascularization following intravitreal bevacizumab (Avastin) injection. Arch Ophthalmol 2006; 124: 1365.

18. Mason JO, Nixon PA, White MF. Intrvitreal injection of bevacizumab (Avastin) as adjuntive treatment of proliferative diabetic retinopathy. Am J Opthalmol 2006; 142: 685688.

19. Nguyen QD, Tatlipinar S, Shah SM, Haller JA, Quinlan E, Sung J, et al. Vascular endotelial growth factor is a critical stimulus for diabetic macular edema. Am J Ophthalmol 2006; 142: 961-969.

20. Chun DW, Heier JS, Topping TM, Duker JS, Banker JM. A pilot study of multiple intravitreal injections of ranibizumab in partients with center involving clinically significant diabetic macular edema. Ophthalmology 2006; 113: 1706-1712.

21. The Macugen Diabetic Retinopathy Study Group. A phase II randomized, double-masked, trial of pegaptanib, an antivascular endothelial growth factor aptamer, for diabetic macular edema. Ophthalmology 2005; 112: 1747-1757.

22. Thomas ER, Wang J, Ege E, Madsen R, Hainsworth DP. Intravitreal triamcinolone acetonide concentration after subtenon injection. Am J Ophthalmol 2006; 142: 860-861.

23. Haritoglou C, Neubauer A, Wolf A, Priglinger S, Strauss $R$, Gandonfer A, et al. Intravitreal bevacizumab (Avastin) therapy for persistent diffuse diabetic macular edema. Retina 2006; 26: 999-1005.

24. Tachi N, Ogino N. Vitrectomy for diffuse diabetic macular edema in cases of diabetic retinopathy. Am J Ophthalmol 1996; 122: 258-260.

25. Otani T, Kishi S. A cotrolled study of vitrectomy for diabetic macular edema. Am J Ophthalmol 2002; 134: 214-219.

26. Avitabile T, Longo A, Reibaldi A. Intravitreal triamcinolone compared with macular laser grid photocoagulation for the treatment of cystoid macular edema. Am J Ophthalmol 2005; 140: 695-702.

\section{NOTA}

* El empleo de triamcinolona intravítrea y de antiangiogénicos está en fase de ensayo clínico. Su uso precisa el consentimiento informado por escrito del paciente y la autorización de la Agencia Española del Medicamento. 